\title{
CORRELAÇÃO ENTRE A PRESENÇA DO CECEIO ANTERIOR E OS TIPOS DE TRESPASSE VERTICAL INTERINCISIVOS NA DENTADURA DECÍDUA
}

\author{
CORRELATION BETWEEN ANTERIOR LISPING AND THE VERTICAL INTERINCISAL TRESPASS IN PRIMARY \\ DENTITION
}

\author{
Nathália Maria Perlato \\ Ana Carla Raphaelli Nahás-Scocate \\ Luciana Badra Jabur \\ Rívea Inês Ferreira \\ Daniela Gamba Garib \\ Karyna Martins do Valle-Corotte
}

\section{RESUMO}

Introdução: Mediante um levantamento epidemiológico em 333 crianças brasileiras, 157 do gênero masculino e 176 do gênero feminino, na faixa etária dos 3 aos 6 anos de idade, matriculadas em uma escola de educação infantil na cidade de São Paulo, foram avaliadas as prevalências e as inter-relaçôes dos diferentes tipos de trespasse vertical interincisivos com o ceceio anterior. Métodos: Todas as crianças envolvidas foram submetidas ao exame clínico da oclusão no plano vertical anterior e à avaliação fonoaudiológica. Além da análise estatística descritiva, abrangendo o cálculo e a tabulação das prevalências dos quatro tipos de trespasse vertical interincisivos (normal, aumentado, nulo e negativo), bem como a da alteraçáo fonoarticulatória estudada, efetuou-se a análise de significância estatística, mediante teste do Qui-quadrado, com nível de significância de 0,05 (95\% de confiança) para a verificação das possíveis relaçốes entre esses dois fatores. Resultados: Os dados quantitativos mostraram as seguintes prevalências: 1 - tipos de trespasse vertical interincisivos: $48,30 \%$ para o normal, $22,50 \%$ para o aumentado, $9,30 \%$ para o nulo e $19,80 \%$ para o negativo; e 2 - presença de ceceio anterior nos tipos de trespasse vertical interincisivos: $42 \%$ para o normal, $12,50 \%$ para o aumentado, $12,50 \%$ para o nulo e $32,90 \%$ para o negativo. Conclusáo: Os resultados da análise estatística mostraram que houve significância para os portadores de ceceio anterior em relação ao trespasse vertical interincisivos negativo e significância estatística para os não portadores de ceceio anterior em relação ao trespasse vertical interincisivos aumentado.

DESCRITORES: Má oclusão • Mordida aberta anterior • Língua • Fonoaudiologia

\section{ABSTRACT}

Introduction: Through a transversal epidemiological study, conducted with 333 Brazilian children, 157 males and 176 females, aged 3 to 6 years old, enrolled in a public preschool in São Paulo city, this study aimed to evaluate the prevalence of the different kinds of vertical interincisal trespass and the relationship between the occlusal aspects and anterior lisping. Metods: All children involved were submitted to a vertical interincisal trespass examination and to a phonoaudiologic evaluation. Besides the descriptive statistical analysis, including the calculation and tabulation of prevalence of the four kinds of interincisal trespass (normal overbite, deep overbite, edge to edge and open bite), the statistical significance was analyzed through the Qui-square test, at a significance level of 0.05 (95\% confidence limits), in order to analyze possible associations between the factors. Results: The quantitative analysis of the data demonstrated the following prevalences: 1 - the different kinds of vertical interincisal trespass: $48.30 \%$ for normal overbite, $22.50 \%$ for deep overbite, $9.30 \%$ for edge to edge and $19.80 \%$ for open bite; and 2- interdental lisping in relationship to the different kinds of vertical interincisal trespass: $42 \%$ for normal open bite, $12.50 \%$ for deep over bite, $12.50 \%$ for edge to edge and $33.30 \%$ for open bite. Conclusion: The statistical analysis of the data demonstrated that there was significance for individuals with anterior lisping in relation to open bite and statistical significance for individuals without anterior lisping in relation to deep overbite.

DESCRIPTORS: Malocclusion • Open Bite • Tongue $\bullet$ Speech.

\footnotetext{
* Graduada pela Universidade Cidade de São Paulo (UNICID). E-mail: nperlato@hotmail.com

** Professora Associada em Ortodontia da Faculdade de Odontologia da Universidade Cidade de São Paulo (UNICID). E-mail: carlanahas@yahoo.com.br

*** Professora Assistente da Disciplina de Ortodontia da Universidade Cidade de São Paulo (UNICID). E-mail: lucianajabur@uol.com.br

**** Professora Associada em Ortodontia da Faculdade de Odontologia da Universidade Cidade de Sáo Paulo (UNICID). E-mail:riveaines@yahoo.com

***** Professora Doutora de Ortodontia, Hospital de Reabilitação de Anomalias Craniofaciais e Faculdade de Odontologia de Bauru da Universidade de São Paulo - USP. E-mail: dgarib@uol.com.br

****** Professora Associada em Ortodontia da Faculdade de Odontologia da Universidade Cidade de São Paulo (UNICID).
} 
Perlato NM, Nahas-Scocate ACR, Jabur LB, Ferreira RI, Garib DG, Valle-Corotte KM. Correlação entre a presença do ceceio anterior e os tipos de trespasse vertical interincisivos na dentadura decídua. Revista de Odontologia da Universidade Cidade de São Paulo 2009 mai-ago; 21(2): 98-103

\section{INTRODUÇÃO}

Os elementos que participam do desenvolvimento e manutenção das características de normalidade da oclusão dentária são numerosos e sensíveis, principalmente em suas inter-relações. Assim sendo, qualquer modificação no mecanismo funcional (respiração, deglutição, fonação e mastigação) poderá alterar a forma, bem como a forma poderá alterar a função, determinando, conseqüentemente, desvios e deformidades morfofuncionais.

Considera-se a língua o órgão mais importante na articulação da fala, devido à sua habilidade em apresentar rápidas mudanças de movimentação e forma. Quando algum tipo de anormalidade impede a sua articulação normal com as estruturas relacionadas (lábios, dentes, alvéolos, palato duro e palato mole), isso pode resultar em um problema de fala.

Alguns pesquisadores concluíram que o organismo humano possui uma tendência natural de compensação postural da musculatura funcional diante de problemas estruturais (más oclusóes), dos fonemas durante a fonoarticulaçáo. É importante destacar que devem ser avaliados criteriosamente os casos em que o organismo não consegue compensar funcionalmente as deficiências morfológicas, para a definição de um correto diagnóstico, plano de tratamento e prognóstico, auxiliando na realização dos tratamentos ortodônticos e evitando possíveis recidivas.

Diversos autores, em seus trabalhos, têm relacionado problemas fonoarticulatórios com diferentes tipos de más oclusóes dentárias, sendo o trespasse vertical interincisivos negativo (mordida aberta anterior) a má oclusão mais associada à presença de ceceio anterior.

Relatou-se também que a persistência dos problemas de fala pode estar relacionada aos insucessos nos tratamentos ortodônticos, sendo que as alteraçóes de fala são consideradas como diagnóstico de um comportamento inadequado de todo o mecanismo funcional e a sua correção pode colaborar para a normalização da oclusão.

O diagnóstico e o tratamento preventivo das alteraçôes morfofuncionais bucais, realizados por uma equipe multidisciplinar envolvendo, além de ortodontistas, cirurgióes dentistas clínicos, médicos pediatras, otorrinolaringologistas, fonoaudiólogos, fisioterapeutas e em algumas vezes psicólogos, são de extrema importância na correção e manutenção dos resultados no relacionamento entre a forma e a função, e suas influências no padrão de crescimento esquelético craniofacial, justificando, dessa maneira, a im- portância do tema abordado nesta pesquisa.

\section{OBJETIVO}

Mediante um levantamento epidemiológico transversal em crianças brasileiras, de ambos os gêneros, na faixa etária dos 3 aos 6 anos de idade, este estudo teve como objetivos:

1. Avaliar a prevalência dos diferentes tipos de trespasse vertical interincisivos na amostra total;

2. Avaliar a prevalência do ceceio anterior em relação aos diferentes tipos de trespasse vertical interincisivos; e

3. Analisar estatisticamente as inter-relaçóes entre os aspectos oclusais e fonoarticulatórios mencionados acima.

\section{MATERIAL E MÉTODOS}

\section{Amostra}

Neste trabalho foram examinados 333 prontuários clínicos pertencentes ao banco de dados do Departamento de Ortodontia da Universidade Cidade de São Paulo - UNICID, referentes a crianças brasileiras, de ambos os gêneros, na faixa etária dos 3 aos 6 anos, matriculadas na Escola Municipal de Educação Infantil (EMEI) Quintino Bocaiúva, no bairro do Tatuapé, na zona leste da cidade de São Paulo. Para atender aos propósitos desta pesquisa, todas as crianças incluídas apresentavam somente dentes decíduos, sem perdas dentárias e com ausência de cáries extensas.

\section{Exame Clínico Odontológico}

Para a avaliação e classificação do trespasse vertical interincisivos, foram utilizados os critérios abaixo:

1. Normal: as bordas incisais dos incisivos superiores recobrem, no máximo, um terço da coroa dos incisivos inferiores (Figura 1);

2. Aumentado (Sobremordida profunda): as bordas incisais dos incisivos superiores recobrem mais de um terço da coroa dos incisivos inferiores (Figura 2);

3. Nulo (Mordida de topo): a distância vertical entre as bordas incisais superiores e inferiores é igual a zero (Figura 3); e

4. Negativo (Mordida aberta anterior): as bordas incisais dos incisivos superiores e inferiores afastam-se verticalmente em direção apical, gerando falta de contato oclusal entre esses dentes (Figura 4). 
Perlato NM, Nahas-Scocate ACR, Jabur LB, Nahás AC, Ferreira RI, Garib DG, Valle-Corotte KM. Correlação entre a presença do ceceio anterior e os tipos de trespasse vertical interincisivos na dentadura decídua. Revista de Odontologia da Universidade Cidade de São Paulo 2009 mai-ago; 21(2): 98-103

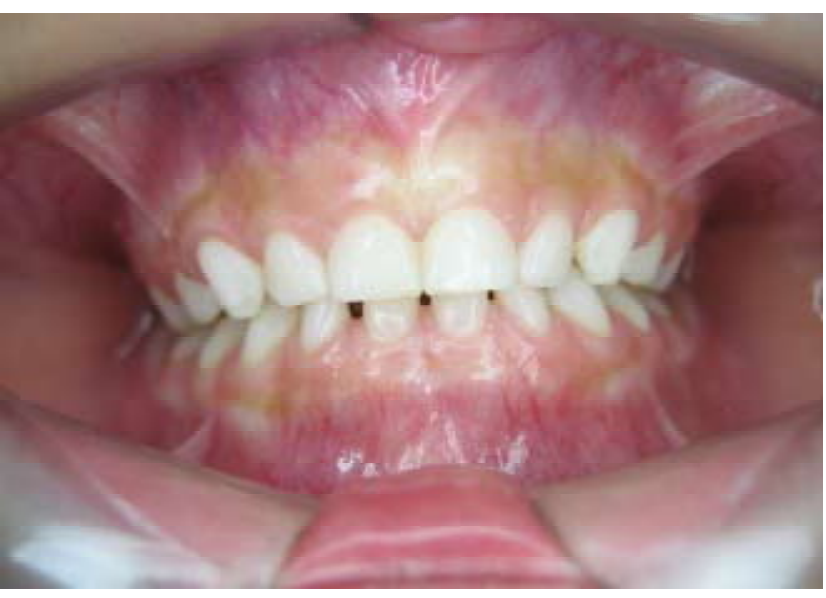

Figura 1 - Trespasse vertical interincisivos normal

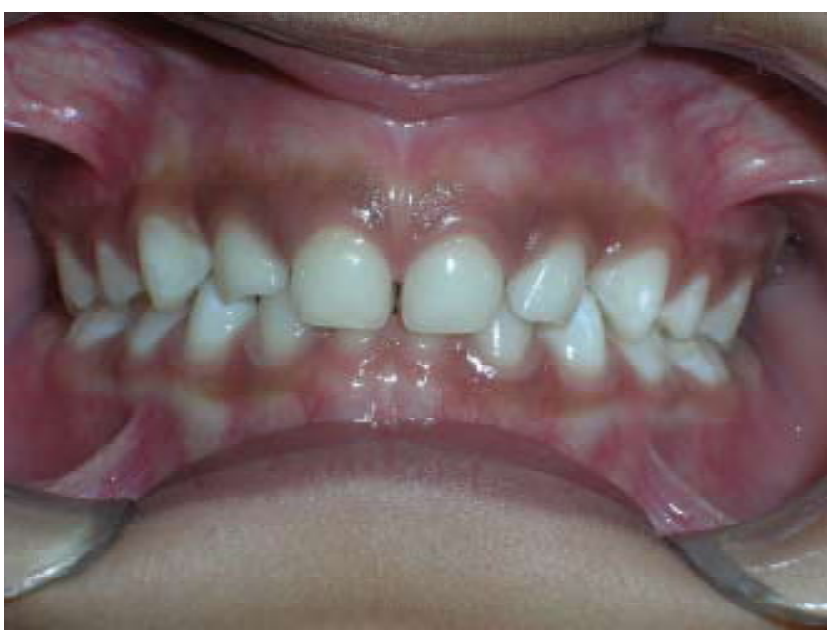

Figura 2 - Trespasse vertical interincisivos aumentado

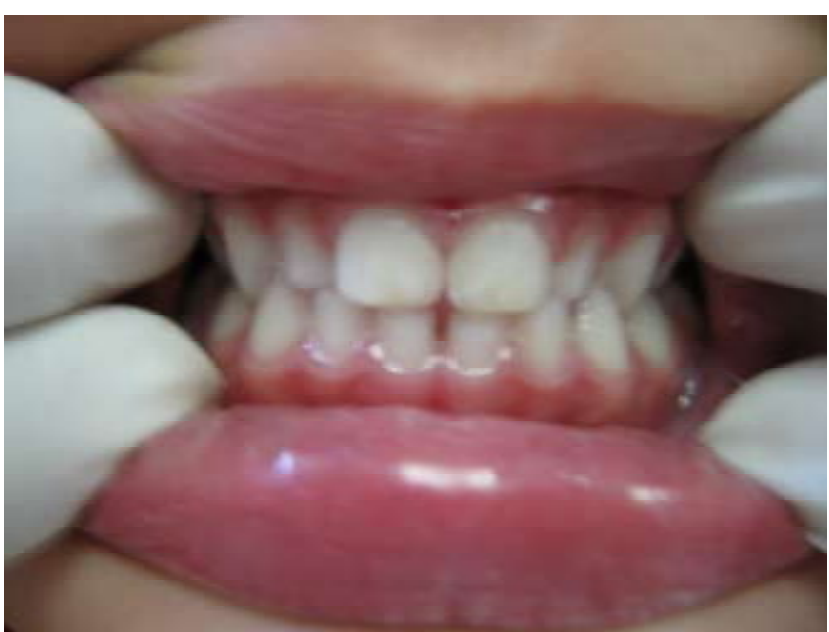

Figura 3 - Trespasse vertical interincisivos nulo

\section{Avaliaçáo Fonoaudiológica}

$\mathrm{Na}$ avaliação da comunicação ora foram executados os seguintes exames: Prova auxiliar - Contexto Pessoal,

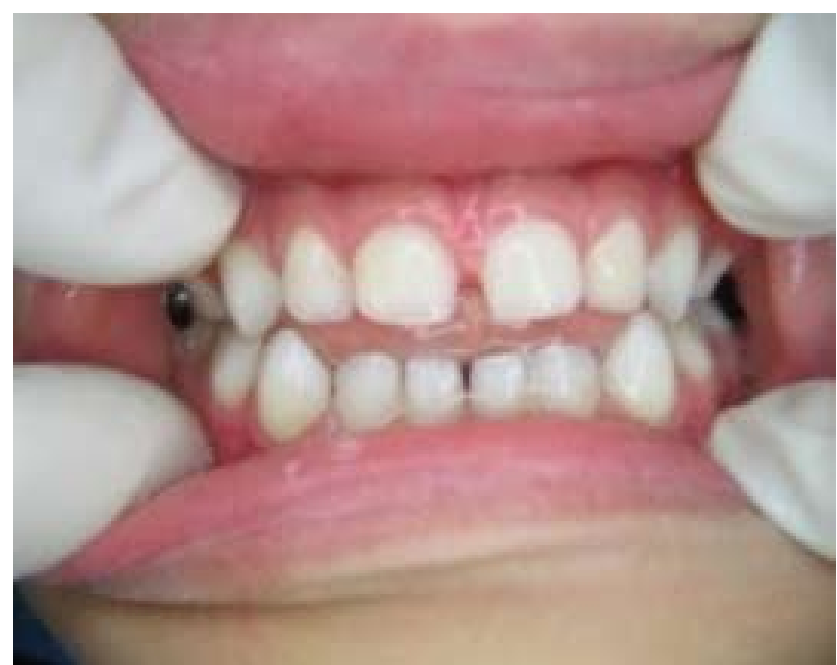

Figura 4 - Trespasse vertical interincisivos negativo

Lista de Palavras - Monossílabos e Dissílabos, Apresentação de Gravuras para Nomeação Espontânea e Avaliação Fonoarticulatória, os quais foram realizados individualmente, em cada criança, no próprio ambiente escolar, sendo eleita uma sala considerada silenciosa, a fim de evitar interferências externas.

\section{RESULTADOS}

Tabela 1 - Prevalência dos diferentes tipos de trespasse vertical interincisivos na amostra estudada.

\begin{tabular}{ccc}
\hline \hline $\begin{array}{c}\text { Tipos de Trespasse Vertical } \\
\text { Interincisivos }\end{array}$ & $\mathrm{n}$ & $\%$ \\
\hline Normal & 161 & 48,3 \\
Aumentado & 75 & 22,5 \\
Nulo & 31 & 9,3 \\
Negativo & 66 & 19,8 \\
Total & 333 & 100,0 \\
\hline \hline
\end{tabular}

Tabela 2 - Prevalência do ceceio anterior em relaçáo aos tipos de trespasse vertical interincisivos da amostra.

\begin{tabular}{|c|c|c|c|c|}
\hline \multirow[t]{2}{*}{$\begin{array}{c}\text { Tipos de trespasse } \\
\text { vertical interincisivos }\end{array}$} & \multicolumn{2}{|c|}{$\begin{array}{l}\text { Crianças com } \\
\text { ceceio anterior }\end{array}$} & \multicolumn{2}{|c|}{$\begin{array}{l}\text { Crianças sem } \\
\text { ceceio anterior }\end{array}$} \\
\hline & $\mathrm{N}$ & $\%$ & $\mathrm{~N}$ & $\%$ \\
\hline Normal & 37 & 42,0 & 124 & 50,6 \\
\hline Aumentado & 11 & 12,5 & 64 & 26,1 \\
\hline Nulo & 11 & 12,5 & 20 & 8,2 \\
\hline Negativo & 29 & 32,9 & 37 & 15,1 \\
\hline Total & 88 & 100,0 & 245 & 100,0 \\
\hline
\end{tabular}


Perlato NM, Nahas-Scocate ACR, Jabur LB, Ferreira RI, Garib DG, Valle-Corotte KM. Correlação entre a presença do ceceio anterior e os tipos de trespasse vertical interincisivos na dentadura decídua. Revista de Odontologia da Universidade Cidade de São Paulo 2009 mai-ago; 21(2): 98-103

Tabela 3 - Resultados da análise estatística, obtidos com o teste do Qui-quadrado, para a obtenção da relação dos diferentes tipos de trespasse vertical interincisivos com o ceceio anterior. Na tabela são mostrados os valores do qui-quadrado, valor-p, e a significância estatística dos resultados.

\begin{tabular}{ccc}
\hline \hline Trespasse Vertical Interincisivos & Ceceio Anterior \\
\hline \multirow{3}{*}{ Normal } & Qui & 1,575 \\
& P & 0,209 \\
& Sig. & NS \\
& Qui & 6,127 \\
Aumentado & P & 0,013 \\
& Sig. & $0,05^{*}$ \\
Nulo & Qui & 0,974 \\
& P & 0,324 \\
& Sig. & NS \\
Negativo & Qui & 11,886 \\
& P & 0,001 \\
& Sig. & $0,001^{*}$ \\
\hline \hline
\end{tabular}

*Diferença estatisticamente significante $(\mathrm{p}<0,05)$.

\section{DISCUSSÃO}

Ainda hoje, pouco se sabe sobre os fatores primários determinantes das más oclusóes. Na maioria das vezes, as características morfofuncionais da oclusão dentária são herdadas no sentido de que haja normalidade, já que os caracteres genéticos geralmente são dominantes. Contudo, os elementos que participam do desenvolvimento e manutenção dessas características de normalidade são numerosos e sensíveis, principalmente em suas inter-relações. Assim sendo, qualquer modificação no mecanismo funcional (respiraçáo, deglutição, fonação e mastigação) poderá alterar a forma, bem como a forma poderá alterar a função, determinando, consequentemente, desvios e deformidades morfofuncionais (Proffit ${ }^{14}$ 1975, Farret ${ }^{2}$ 1998, Marchesan ${ }^{12}$ 2000). Vale lembrar que segundo Graber $^{3}$ (1974), apesar de ser o tecido ósseo um dos mais duros do corpo humano, ele é um dos mais plásticos e que mais responde às forças geradas durante a função.

No que se referem à fala, muitas pesquisas não obtiveram resultados conclusivos na tentativa de relacionar as alterações fonoarticulatórias com as más oclusóes e desvios nas dimensões das estruturas bucais. Contudo, tais relaçôes não foram totalmente descartadas, havendo concordância entre os autores de que mais estudos devem ser realizados, visando um maior esclarecimento dessa relação (Coleman e Gullikson ${ }^{1}$ 1971, Proffit $^{14}$ 1975, Laine ${ }^{8}$ 1986, Kellum et al. ${ }^{6} 1994$, Tomita et al. ${ }^{17}$ 2000). Considera-se a língua o órgão mais importante na articulação da fala, devido à sua habilidade em apresentar rápidas mudanças de movimentação e forma. Quando algum tipo de anormalidade impede a sua articulação normal com as estruturas relacionadas (lábios, dentes, alvéolos, palato duro e palato mole), isso pode resultar em uma alteração de fala (Coleman e Gullikson ${ }^{1}$ 1971, Kenth e Schaaf 5 1982).

Diversos autores, em seus trabalhos, têm relacionado desvios fonoarticulatórios com diferentes tipos de más oclusóes dentárias (Coleman e Gullikson ${ }^{1}$ 1971, Proffit $^{14}$ 1975, Klechak et al. ${ }^{7}$ 1976, Guayet al. ${ }^{4} 1978$, Kenth e Schaaf ${ }^{5}$ 1982, Laine ${ }^{9}$ 1987, Laine et al. ${ }^{11} 1987$, Zaldívar et al. ${ }^{18} 1987$, Pahkala et al. ${ }^{13} 1995$, Marchesan ${ }^{12}$ 2000), sendo o trespasse vertical interincisivos negativo (mordida aberta anterior) a má oclusão mais associada à presença de ceceio anterior e projeção lingual anterior na emissão dos fones linguoalveolares (Coleman e Gullikson $^{1}$ 1971, Klechak et al. ${ }^{7}$ 1976, Guay ${ }^{4}$ 978, Kenth e Schaaf ${ }^{5}$, Laine ${ }^{9}$ 1987, Laine et al. ${ }^{11}$ 1987, Zaldívar et al. ${ }^{19}$ 1987, Laine ${ }^{10}$ 1992, Pahkala et al. ${ }^{13} 1995$, Marchesan ${ }^{12}$ 2000, Sahad et al. ${ }^{16}$ 2008).

O número total da amostra foi considerado um número expressivo, sendo superior ao das amostras utilizadas em outros trabalhos realizados na mesma linha de pesquisa (Klechak et al. ${ }^{7}$ 1976, Guay et al. ${ }^{4} 1978$; Zaldívar et al. ${ }^{19}$ 1987, Laine 1987 , Qvarnstrom et al. ${ }^{15}$ 1993, Pahkala et al. ${ }^{13}$ 1995), gerando maior credibilidade e confiabilidade aos resultados do atual estudo.

Depreende-se dos resultados apresentados nesta pesquisa, bem como de outros trabalhos aqui citados, que há uma relação direta entre as alterações fonoarticulatórias estudadas e o trespasse vertical negativo e diminuição das alteraçóes de fala na presença do trespasse vertical interincisivos aumentado. Contudo, é incorreto responsabilizar somente um tipo de má oclusão como sendo o causador de alterações fonoarticulatórias, pois pesquisas anteriores relatam que, normalmente, a combinação de vários tipos de más oclusóes presentes em um único indivíduo, aliados ao padrão facial genético desfavorável, presença de hábitos bucais deletérios, o tônus da musculatura bucal alterado, o modo de respiração e o tipo de deglutição, aumentam, em grande número, a probabilidade do surgimento de desvios fonéticos (Laine ${ }^{10}$ 
Perlato NM, Nahas-Scocate ACR, Jabur LB, Nahás AC, Ferreira RI, Garib DG, Valle-Corotte KM. Correlação entre a presença do ceceio anterior e os tipos de trespasse vertical interincisivos na dentadura decídua. Revista de Odontologia da Universidade Cidade de São Paulo 2009 mai-ago; 21(2): 98-103

1992, Marchesan ${ }^{12}$ 2000). Até mesmo porque 54,5\% das crianças com ceceio anterior exibiam trespasse vertical interincisivos normal e aumentado, demonstrando claramente a existência de outros fatores etiológicos envolvidos na gênese deste distúrbio fonoarticulatório.

Apesar de os resultados indicarem uma relação positiva do ceceio anterior com o trespasse vertical interincisivos negativo (mordida abeta anterior), a análise das características oclusais deve ser complementada com o estudo de todas as estruturas orofaciais associadas e do padrão facial para se avaliar a relação existente entre as alterações de fala e as más oclusões. Para isso, sugere-se a utilização de métodos auxiliares de diagnóstico, como a cefalometria, com o objetivo de se determinar o padrão facial genético, além da análise e avaliação das estruturas orofaciais e suas dimensóes por meio de modelos de estudo, contribuindo dessa maneira para resultados mais fidedignos.

\section{CONCLUSÕES}

A análise estatística dos dados mostrou que:

- $\mathrm{Na}$ amostra total, a prevalência dos diferentes tipos de trespasse vertical interincisivos foi de: $48,3 \%$ para o normal, $22,5 \%$ aumentado, 9,3\% nulo e 19,8\% negativo;

- A prevalência do ceceio anterior em relação aos diferentes tipos de trespasse vertical interincisivos foi de: $42 \%$ para o normal, $12,5 \%$ aumentado, $12,5 \%$ nulo e $32,9 \%$ negativo; e

- Houve significância para os portadores de ceceio anterior em relação ao trespasse vertical interincisivos negativo e houve significância para os não portadores de ceceio anterior em relação ao trespasse vertical interincisivos aumentado.

\section{REFERÊNCIAS}

1. Coleman RO, Gullikson JS. Speech problems in children. J Denta Child 1971 Nov-Dec 28(6): 381-4.

2. Farret MMB, Jurach EM, Brandão L, Moraes DCF, Brandão SRS, Santos SL. Relationship between malocclusion and fonoarticulatory disorders. Int J Orofacial Myology. 1998; 24: 20-6.

3. Graber TM. Ortodoncia. Teorica y Pratica. Tradução: Gracia JL. $3^{a}$ Ed. México Edit. Interamericana. 1974.

4. Guay AH, Maxwell DL, Beecher R. A radiographic study of tongue posture at rest and during the phonation of /s/ in class III malocclusion. Angle Orthod. 1978 Jan 48(1): 10-22.

5. Kent K, Schaaf NG. The effects of Dental Abnormalities on speech production. Quintessence Inter Dent Dig. 1982 Dec 13(12): 1353-62.

6. Kellum GD, Gross, AM Hale ST, Eiland S, Williams C. Thombsucking as related to placement and acoustic of $/ \mathrm{s}, \mathrm{z} /$ and lingual rest postures. Int J Orofacial Myology. 1994. 20: 4-9.

7. Klechak TL, Bradley DP, Warren DW. Anterior open bite and oral port constriction. Angle Orthod. 1976 46(3): 232-42.
8. Laine T. Articulatory disorders in speech as related to size of the alveolar arches. Euro J Orthod. 1986 Aug 8(3): 192-7.

9. Laine T. Associations between articulatory disorders in speech and occlusal anomalies. Euro J Orthd. 1987 May 9(2): 144-50.

10. Laine T. Malocclusion traits and articulatory components of speech. Euro J Orthod. 1992 Aug 14(4): 302-9

11. Laine T, Jaroma M, Linnasalo AL. Relationships between interincisal occlusion and articulatory components of speech. Folia Phoniat. 1987 39(2): 78-86.

12. Marchesan IQ. The speech pathology treatment with alterations os a stomatgnathic system. Int J Orofacial Myology. 2000 Nov 26(5): 5-12.

13. Pahkala R, Laine T, Narhi M. Associations among different orofacial dysfunctions in 9-11 year-olds. Euro J Orthod. 1995 Dec 17(6): 497-506.

14. Proffit W. Muscle pressures and tooth position: North American Whites and Australian Aborigenes. Angle Orthod. 1975; 45 (1): 1-11. 
Perlato NM, Nahas-Scocate ACR, Jabur LB, Ferreira RI, Garib DG, Valle-Corotte KM. Correlação entre a presença do ceceio anterior e os tipos de trespasse vertical interincisivos na dentadura decídua. Revista de Odontologia da Universidade Cidade de São Paulo 2009 mai-ago; 21(2): 98-103

15. Qvarnström M, Jaroma M, Laine T. Accuracy of articulatory movements of speech in a group of firstgraders. Folia Proniat. 1993; 45: 214-22

16. Sahad MG, Nahas ACR, Scavone-Júnior H, Jabur LB, Guedes-Pinto E. Vertical interincisal trespass assessment in children with speech disorders. Braz Oral Res. 2008. 22(3): 247-51.

17. Tomita NE, Bijella VT, Franco LJ. Relação entre hábitos bucais e má oclusão em pré-escolares. Ver Saúde Pública. 2000 Jun 34(3): 299-303.
18. Zaldívar CV, Rodrigues JB, Pérez EF. Las maloclusiones y su relación con los transtornos del lenguaje. Parte I. Rev Cubana Estomatol. 1987; 24(2): 135-140.

19. Zaldívar CV, Rodrigues JB, Pérez EF. Las maloclusiones y su relación con los transtornos del lenguaje. Parte II. Rev Cubana Estomatol. 1987; 24(2): 141-7.

Recebido em: 25/08/2008

Aceito em: 24/03/2009 